\title{
Accounting for Antenna in Half-Space Fresnel Coefficient Estimation
}

\author{
A. D'Alterio and R. Solimene \\ Dipartimento di Ingegneria dell'Informazione, Seconda Università di Napoli, Via Roma 29, 81031 Aversa, Italy \\ Correspondence should be addressed to A. D’Alterio, antonietta.dalterio@unina2.it
}

Received 27 April 2012; Accepted 14 June 2012

Academic Editor: Francesco Soldovieri

Copyright (C 2012 A. D’Alterio and R. Solimene. This is an open access article distributed under the Creative Commons Attribution License, which permits unrestricted use, distribution, and reproduction in any medium, provided the original work is properly cited.

The problem of retrieving the Fresnel reflection coefficients of a half-space medium starting from measurements collected under a reflection mode multistatic configuration is dealt with. According to our previous results, reflection coefficient estimation is cast as the inversion of linear operator. However, here, we take a step ahead towards more realistic scenarios as the role of antennas (both transmitting and receiving) is embodied in the estimation procedure. Numerical results are presented to show the effectiveness of the method for different types of half-space media.

\section{Introduction}

Subsurface imaging problem is relevant in several applicative contexts that range from geophysical to civil engineering applications [1].

In this framework, regardless of the imaging algorithm one may want to use, the knowledge of soil parameters is necessary in order to obtain properly focused images and free from artifacts proliferation [2].

By contrast, in realistic scenarios, such parameters are generally unknown or, at best, known with some degree of uncertainty. Therefore, a soil parameter estimation procedure must be run before imaging.

Many procedures for estimating soil parameters are widespread in the literature. Reflectometry methods are very common [3]. There are methods which rely on fitting the moveout of hyperbolic diffraction pattern or measure travel time to a scatterer buried at known depth [4]. Other methods exploit different offset data and perform velocity or amplitude analysis to gather soil properties $[5,6]$. Iterative imaging instead identifies soil parameters as those which return the more focalized reconstruction of a cooperative target [7]. Finally, further methods first retrieve reflection coefficient and then infer the soil properties by minimizing a nonlinear cost function by using optimization procedure [8].

Most of the methods quoted above require far zone approximation, so that asymptotic ray approximation works and generally assume soil as a homogeneous (at least transversally) half space. Moreover, time domain data, equivalently multifrequency data, are employed. In particular, this requires dealing with a non-linear inversions when the reflection coefficient is used to infer soil properties [8]. As well known, non-linear inversion are generally computationally demanding and can suffer from reliability problems due to the occurrence of false solutions. In these cases, one can take advantage from a priori information about the soil which allows to reduce the number searched for unknowns. However, this entails that soil dispersive law must be a priori known.

Determining soil electromagnetic parameters is a mandatory step to achieve soil analysis in order to assess, for example, water content and in general material composition. However, this is not necessarily hold for imaging. Indeed, what is really needed to obtain properly focused image of buried scatterers is the relevant Green's function. Under half-space homogenous assumption for soil (which is an assumption commonly used, as can be argued from previous 
references), Green's function can be directly determined from the half-space Fresnel reflection coefficients [9]. Of course, from the Fresnel coefficients soil properties can be inferred as well, but as said above this step is not required for imaging. However, it is remarked that following this new perspective requires to retrieve the reflection coefficients not only as a function of the time frequency but also as a function of the spatial frequencies (i.e., for different angles of incidence).

Recently, in [10] a new estimation method, which achieves such a task, was proposed. There, proximal GPR measurements, collected at different positions, were exploited to develop an estimation method based on multiview (multi-offsset) information. Therefore, far-zone approximation were no longer necessary. More precisely, the problem is cast as the inversion of a linear integral operator linking the reflected field and the Fresnel reflection coefficients. When such a problem is solved, an estimation of the reflection coefficient is available for different angle of incidence but for a fixed-time frequency. Therefore, the procedure must be run for each adopted frequency. However, this inconvenient is not too critical, as to image shallowly buried scatterers the number of necessary frequency can be not so high $[11,12]$ and is traded off by the advantage that soil dispersive laws are not required.

The method presented in [10] was first developed and numerically validated by considering two-dimensional scalar cases. Then, the procedure was extended to threedimensional cases where reflection coefficient is indeed a matrix. Therefore, in that cases the problem was cast as the inversion of a linear integral operator where the relevant unknowns (i.e., the Fresnel coefficients) appear embedded within a dyad. As shown in [13], depending on antennas' polarization, this gives rise to different strategies for achieving the solution.

However, in that contribution, reflected field measurements were considered as data, instead, in practical cases, what is actually measured is the voltage signal and not the field. Hence, the estimation scheme must account also for the role of the receiving antenna. This is just the further step towards a more realistic scenario done in this paper.

The plan of the paper is the following. In Section 2, we describe the adopted measurement configuration and introduce the problem mathematical formulation. In Section 3, the procedure for retrieving the Fresnel reflection coefficients is introduced, whereas in Section 4 numerical results are reported for different types of soil. Conclusions follow.

\section{Geometry and Problem Formulation}

The scattering problem depicted in Figure 1 is of concern.

The scenario consists of a two-layered medium separated by a planar interface at $z=0$. The upper layer is assumed to be the free-space with $\varepsilon_{0}$ and $\mu_{0}$ being its dielectric permittivity and magnetic permeability, respectively. The

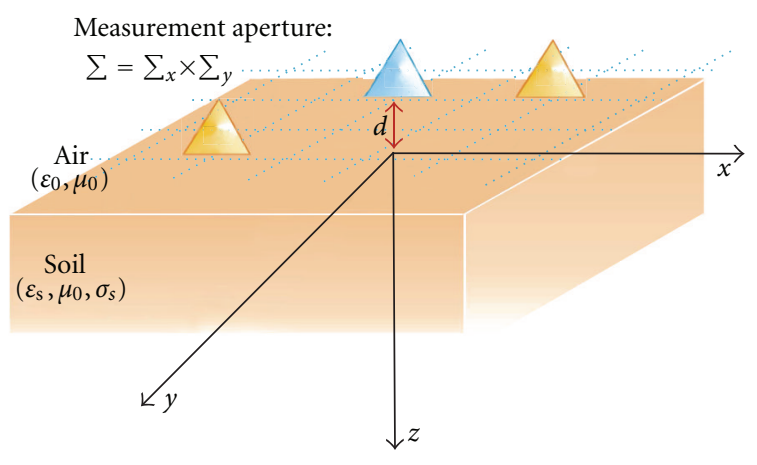

Figure 1: Geometry of the problem.

lower half-space is representative of the soil which is assumed nonmagnetic (i.e., its magnetic permeability is equal to the one of free space) and homogeneous with a relative dielectric permittivity and electric conductivity, denoted as $\mathcal{E}_{s}$ and $\sigma_{S}$, respectively.

Accordingly, in the frequency domain the dielectric permittivity is actually a complex function which depends on the angular frequency $\omega$ and thus the relative equivalent permittivity is given by $\varepsilon_{\text {eq }}(\omega)=\varepsilon_{S}(\omega)-j \sigma_{S} / \omega$.

The transmitting antenna is located in the upper halfspace at height $d$ from the air/soil interface in $P=$ $\left(x_{s}, y_{s},-d\right)$. It is assumed that it is far enough so that the effect of the soil on the antenna radiation properties is negligible or a proper gating procedure, as suggested in [14], is employed before the estimation takes place.

We consider a multistatic measurement configuration where the reflected field is collected over a set of different positions taken over the observation domain $\Sigma=\Sigma_{x} \times \Sigma_{y}$ which is parallel to the air/soil interface and located at the same height as the source. $\Sigma_{x}=\left[-X_{O}, X_{O}\right]$ and $\Sigma_{y}=$ $\left[-Y_{O}, Y_{O}\right]$ are the extent of the observation domain along the $x$ - and $y$-axes, respectively.

As discussed in the Introduction, we aim at retrieving Fresnel reflection coefficients from reflected field measurements. Moreover, this must be achieved for all the relevant spatial spectral harmonics as these are necessary for Green's function determination for a subsequent imaging stage.

Accordingly, it is natural to express the link between the reflected field, $\underline{E}_{r}\left(x_{O}, y_{O}, k_{0}\right)$, and the reflection coefficients, in terms of plane-wave spectrum notation, that is,

$$
\begin{aligned}
& \underline{E}_{r}\left(x_{O}, y_{O}, k_{0}\right) \\
& =\frac{1}{(2 \pi)^{2}} \iint_{D} \underline{\Gamma}\left(k_{x}, k_{y}, k_{0}\right) \underline{f}_{S}\left(k_{x}, k_{y}, k_{0}\right) \\
& \quad \times \exp \left[-j k_{x}\left(x_{O}-x_{S}\right)\right] \exp \left[-j k_{y}\left(y_{O}-y_{S}\right)\right] \\
& \quad \times \exp \left(-j 2 k_{z} d\right) d k_{x} d k_{y},
\end{aligned}
$$


where $\underline{\underline{\Gamma}}\left(k_{x}, k_{y}, k_{0}\right)$ is the dyadic reflection coefficient at the air/soil interface [13]:

$$
\begin{aligned}
& \underline{\underline{\Gamma}}\left(k_{k}, k_{y}, k_{0}\right) \\
& =\left[\begin{array}{ccc}
\left(\Gamma_{\mathrm{TE}} \frac{k_{y}^{2}}{k_{t}^{2}}+\Gamma_{\mathrm{TM}} \frac{k_{x}^{2}}{k_{t}^{2}}\right) & \left(\Gamma_{\mathrm{TM}} \frac{k_{x} k_{y}}{k_{t}^{2}}-\Gamma_{\mathrm{TE}} \frac{k_{x} k_{y}}{k_{t}^{2}}\right) & 0 \\
\left(\Gamma_{\mathrm{TM}} \frac{k_{x} k_{y}}{k_{t}^{2}}-\Gamma_{\mathrm{TE}} \frac{k_{x} k_{y}}{k_{t}^{2}}\right) & \left(\Gamma_{\mathrm{TE}} \frac{k_{x}^{2}}{k_{t}^{2}}+\Gamma_{\mathrm{TM}} \frac{k_{y}^{2}}{k_{t}^{2}}\right) & 0 \\
0 & 0 & -\Gamma_{\mathrm{TM}}
\end{array}\right]
\end{aligned}
$$

$\Gamma_{\mathrm{TE}}$ and $\Gamma_{\mathrm{TM}}$ are the Fresnel reflection coefficients, $\underline{f}_{S}=$ $\left(f_{x}, f_{y}, f_{z}\right)$ is the source plane-wave spectrum, $k_{x}, \bar{k}_{y}, k_{z}$ are the wavenumber components corresponding to $x, y, z$, respectively, and $k_{t}^{2}=k_{x}^{2}+k_{y}^{2}$. Finally, $k_{0}=\omega \sqrt{\varepsilon_{0} \mu_{0}}$ is the free-space wavenumber.

Note that in (1) the spectral integration interval $D$ corresponds to the minimum square enclosing the circle of radius $k_{0}$ which accounts for the so-called "visible domain."

Equation (1) represents the link to be inverted in order to retrieve reflection coefficients. In particular, it should be solved for $\Gamma_{\mathrm{TE}}$ and $\Gamma_{\mathrm{TM}}$ which appear embodied within $\underline{\underline{\Gamma}}$.

A way to achieve such a task has been described in [13]. That paper reports promising results but has to be meant as a proof of principle. Indeed, the inversion of (1) relies on field measurements whereas what can be actually measured is antenna output voltage. In other words, the role of receiving antenna has to be accounted for and (1) properly modified.

To this end, here, a simple truncated waveguide is considered as transmitting and receiving antenna. In particular, the source point $x_{s}, y_{s},-d$ and the observation point $x_{O}, y_{O},-d$ correspond to the centre of the antenna apertures. For convenience, we also introduce a local reference frame $x^{\prime}, y^{\prime}, z^{\prime}$ (see Figure 2) to address points within the antenna aperture for each antenna position.

By neglecting finite aperture effects and by assuming that the guide is matched, then the field at the antenna terminal section $z^{\prime}=0$ (for each receiving antenna positions) is just the incoming field reflected by the soil $\underline{E}_{r}\left(x^{\prime}, y^{\prime}, 0\right)$.

Hence, we can write the "voltage" at $z^{\prime}=0$ associated to the waveguide fundamental mode as [15]

$$
V_{10}^{\mathrm{TE}}(0)=\iint_{S} \underline{E}_{r}\left(x^{\prime}, y^{\prime}, 0\right) \times \underline{h}_{10}^{\mathrm{TE}} \cdot \hat{i}_{z^{\prime}} d S
$$

In particular, if the working frequency is properly chosen and $L$ sufficiently greater than the wavelength (see Figure 2 ), this is the only relevant contribution at the reference plane in $z^{\prime}=$ $-L$ as high-order modes are in cutoff.

Accordingly, the voltage at the reference section writes as

$$
V=V_{10}^{\mathrm{TE}}(0) \exp \left(-i k_{z}^{\prime} L\right)
$$

where $k_{z}^{\prime}=\sqrt{k_{0}^{2}-(\pi / a)^{2}}$ being the $z$-component of the waveguide wavevector.

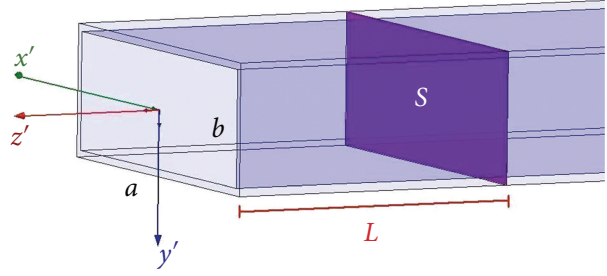

Figure 2: Truncated waveguide antenna.

Eventually, the relationship upon which the estimation procedure is based is obtained by inserting (1) in (4); that is

$$
\begin{aligned}
& V\left(x_{O}, y_{O}, k_{0}\right) \\
& =\exp \left(-i k_{z}^{\prime} L\right) \\
& \times \iint_{S} \frac{1}{(2 \pi)^{2}} \iint_{D} \underline{\Gamma}\left(k_{x}, k_{y}, k_{0}\right) \\
& \quad \times \underline{f}_{S}\left(k_{x}, k_{y}, k_{0}\right) \times \underline{h}_{10}^{\mathrm{TE}} \\
& \quad \times \exp \left[-j k_{x}\left(x^{\prime}+x_{O}-x_{S}\right)\right] \\
& \quad \times \exp \left[-j k_{y}\left(y^{\prime}+y_{O}-y_{S}\right)\right] \\
& \quad \times \exp \left(-j 2 k_{z} d\right) d k_{x} d k_{y} d S .
\end{aligned}
$$

\section{Estimation Procedure}

In this section we introduce the procedure to estimate $\Gamma_{\mathrm{TE}}$ and $\Gamma_{\mathrm{TM}}$ (embedded in the coefficients of $\Gamma$ ) starting from voltage measurements taken at a grid of points over $\Sigma$.

To this end, different inversion strategies can be employed to invert (5) and de-embed $\Gamma_{\mathrm{TE}}$ and $\Gamma_{\mathrm{TM}}$ from $\underline{\underline{\Gamma}}$ [13]. Basically, by changing the polarization of the transmitting and/or the receiving antennas, a couple of linear integral equations are obtained whose solution gives two different parameters of the reflection matrix (2). Hence, from these two independent equations, the Fresnel coefficients are obtained.

Here, we adopt the strategy depicted in Figure 3. In particular, the two needed voltage measurements (for each observation point) are obtained by considering first the transmitting and receiving antennas polarized along the $x$ axis. Accordingly, (5) particularizes as

$$
\begin{aligned}
& V_{1}\left(x_{O}, y_{O}, k_{0}\right) \\
& \begin{aligned}
&=\frac{1}{(2 \pi)^{2}} \sqrt{\frac{2}{a b}} \iint_{D} \Gamma_{11}\left(k_{x}, k_{y}, k_{0}\right) f_{x}^{2}\left(k_{x}, k_{y}, k_{0}\right) \\
& \times \exp \left[-j k_{x}\left(x_{O}-x_{S}\right)\right] \\
& \times \exp \left[-j k_{y}\left(y_{O}-y_{S}\right)\right] \exp \left(-j 2 k_{z} d\right) \\
& \times \exp \left(-j k_{z}^{\prime} L\right) d k_{x} d k_{y},
\end{aligned}
\end{aligned}
$$




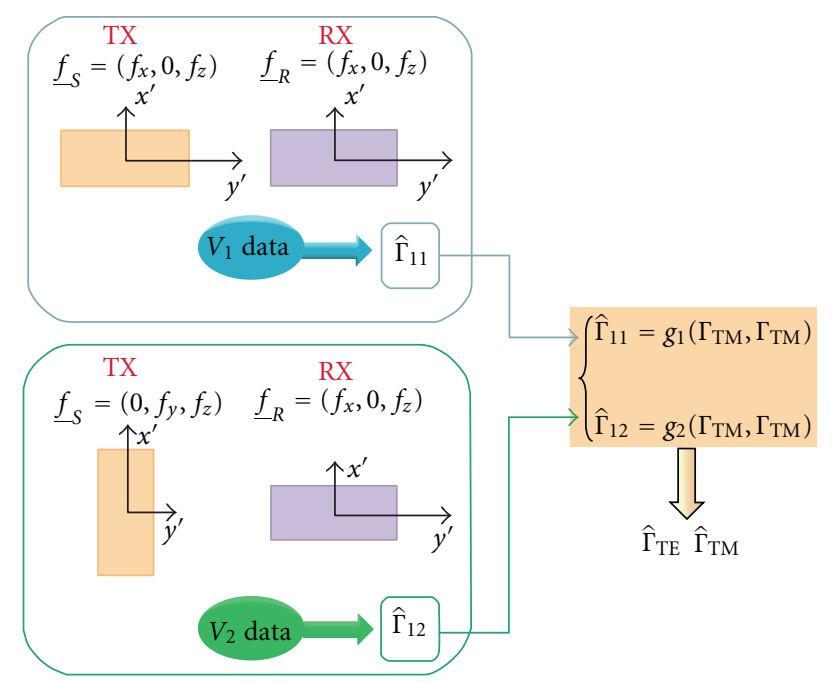

FIgURE 3: Illustrating the overall estimation procedure.

where we considered that $\underline{f}_{S}=\underline{f}_{R}=\left(f_{x}, 0, f_{z}\right), \Gamma_{11}$ is the $(1,1)$ entry of $\underline{\Gamma}, a$ and $b$ are the guide transverse dimensions (with $a>b$ ), and $\underline{k}_{t}=\left(k_{x}, k_{y}, 0\right)$.

The second equation is obtained by rotating the transmitting antenna of 90 degrees so that it results to be polarized along the $y$-axis; that is, $\underline{f}_{S}=\left(0, f_{x}, f_{z}\right)$. In this case (5) becomes

$$
\begin{aligned}
& V_{2}\left(x_{O}, y_{O}, k_{0}\right) \\
& =\frac{1}{(2 \pi)^{2}} \sqrt{\frac{2}{a b} \iint_{D}} \Gamma_{12}\left(k_{x}, k_{y}, k_{0}\right) f_{x}^{2}\left(k_{x}, k_{y}, k_{0}\right) \\
& \times \exp \left[-j k_{x}\left(x_{O}-x_{S}\right)\right] \\
& \times \exp \left[-j k_{y}\left(y_{O}-y_{S}\right)\right] \\
& \times \exp \left(-j 2 k_{z} d\right) \\
& \times \exp \left(-j k_{z}^{\prime} L\right) d k_{x} d k_{y}
\end{aligned}
$$

where now the term $\Gamma_{12}$ of $\underline{\underline{\Gamma}}$ is involved.

We have now a pair of integral equations whose inversion returns an estimation for $\Gamma_{11}(\cdot)$ and $\Gamma_{21}(\cdot)$, that is, $\widehat{\Gamma}_{11}(\cdot)$ and $\widehat{\Gamma}_{21}(\cdot)$, from which, according to $(2)$, the Fresnel reflection coefficients can be obtained via algebraic relations as

$$
\begin{gathered}
\hat{\Gamma}_{\mathrm{TE}}\left(k_{x}, k_{y}, k_{0}\right)=\frac{\Gamma_{1}\left(k_{x}, k_{y}, k_{0}\right)-k_{x}^{2} \Gamma_{2}\left(k_{x}, k_{y}, k_{0}\right)}{k_{t}^{2}}, \\
\hat{\Gamma}_{\mathrm{TM}}\left(k_{x}, k_{y}, k_{0}\right)=\frac{\Gamma_{1}\left(k_{x}, k_{y}, k_{0}\right)+k_{y}^{2} \Gamma_{2}\left(k_{x}, k_{y}, k_{0}\right)}{k_{t}^{2}},
\end{gathered}
$$

where $\Gamma_{1}(\cdot)=k_{t}^{2} \widehat{\Gamma}_{11}(\cdot), \Gamma_{2}(\cdot)=\left(k_{t}^{2} / k_{x} k_{y}\right) \widehat{\Gamma}_{21}(\cdot), \widehat{\Gamma}_{\mathrm{TE}}(\cdot)$ and $\widehat{\Gamma}_{\mathrm{TM}}(\cdot)$ are the estimated Fresnel reflection coefficients.

In this plan, the inversion of the two integral equations (6) and (7) is crucial in order to get a reasonable reflection coefficient estimations. In particular, since such operators are compact, the corresponding inversion is an ill-posed inverse problem [16]. Accordingly, a suitable inversion scheme has to be adopted to establish a proper compromise between accuracy and stability against uncertainties. To cope with this point, inversions are achieved by exploiting the truncated singular value decomposition (SVD) method [16]. Details concerning the singular value threshold $N_{T}$, the number of required measurements and unknown representation (to obtain discrete counterparts of (6) and (7)), and noise propagation can be found in [13]. Here, we report only the relevant equations. On denoting as $\left\{u_{n}, v_{n}, \sigma_{n}\right\}_{n=0}^{\infty}$ the singular system of one of the involved integral operators, where $u_{n}, v_{n}$, and $\sigma_{n}$ are the $n$th left singular function, the $n$th right singular function, and the $n$th singular value, respectively, the corresponding solutions can be expressed as

$$
\begin{aligned}
& \hat{\Gamma}_{11}\left(k_{x}, k_{y}, k_{0}\right)=\sum_{n=0}^{N_{T}} \frac{\left\langle V_{1}, v_{n}\right\rangle}{\sigma_{n}} u_{n}, \\
& \hat{\Gamma}_{21}\left(k_{x}, k_{y}, k_{0}\right)=\sum_{n=0}^{N_{T}} \frac{\left\langle V_{2}, v_{n}\right\rangle}{\sigma_{n}} u_{n} .
\end{aligned}
$$

We end this section by remarking that (8) allows to retrieve the Fresnel coefficient as a function of the spectral angular variables and for a fixed frequency. When more frequency are needed, as usually occurs during imaging, the procedure outlined above must be repeated for each adopted frequency.

\section{Numerical Results}

In this section, we report some numerical reconstructions obtained by exploiting synthetic data with the aim to assess the performance achievable by the estimation procedure. The source is located at $\underline{r}_{S}=(0,0,-d)$, with $d=0.1 \mathrm{~m}$, whereas receiving antenna span a grid of $N_{x} \times N_{y}=20 \times$ 20 points taken uniformly over the observation domain $\Sigma=\left[-X_{O}, X_{O}\right] \times\left[-Y_{O}, Y_{O}\right]=[-1,1] \times[-1,1] \mathrm{m}^{2}$ and $\mathrm{a}$ frequency $f=1.5 \mathrm{GHz}$ is adopted.

The waveguide transverse dimensions are $a=0.16 \mathrm{~m}, b=$ $0.08 \mathrm{~m}$ whereas the reference plane is fixed at $h=0.1 \mathrm{~m}$ from the aperture.

We consider three kinds of soil addressed for convenience, in the following, as soil 1, soil 2, and soil 3. More in detail, soil 1 is characterized by $\varepsilon_{\text {eq }}=4 \varepsilon_{0}$, hence describes a nondissipative as well as nondispersive medium; soil 2 accounts for Ohmic losses, hence $\varepsilon_{\mathrm{eq}}=4 \varepsilon_{0}-j(0.1 / \omega)$; finally soil 3 considers both Ohmic losses and dielectric hysteretic behaviours so that $\varepsilon_{\mathrm{eq}}(\omega) / \varepsilon_{0}=\varepsilon_{\infty}-j\left(\sigma_{S} / \omega \varepsilon_{0}\right)+\left(\left(\varepsilon_{S}-\varepsilon_{\infty}\right) /(1+\right.$ $\left.j \omega \tau_{e}\right)$ ) with $\varepsilon_{\infty}=4, \varepsilon_{S}=16, \sigma_{S}=0.1 \mathrm{~S} / \mathrm{m}$, and $\tau_{e}=64 \mathrm{~ns}$. Moreover, in order to test the procedures for data corrupted by noise, some examples are obtained by adding, to both voltage data, a complex zero mean white Gaussian noise $n$ so as to have a $\mathrm{SNR}=20 \log \left(\left\|V_{1,2}\right\| /\|n\|\right)=30 \mathrm{~dB}$.

The reconstructions corresponding to the three types of soil are reported in Figures 4 and 5, Figures 6 and 7, Figures 8 and 9 , respectively. In those figures, for comparison purposes, beside being noiseless and noisy estimations also actual Fresnel reflection coefficients are shown. 

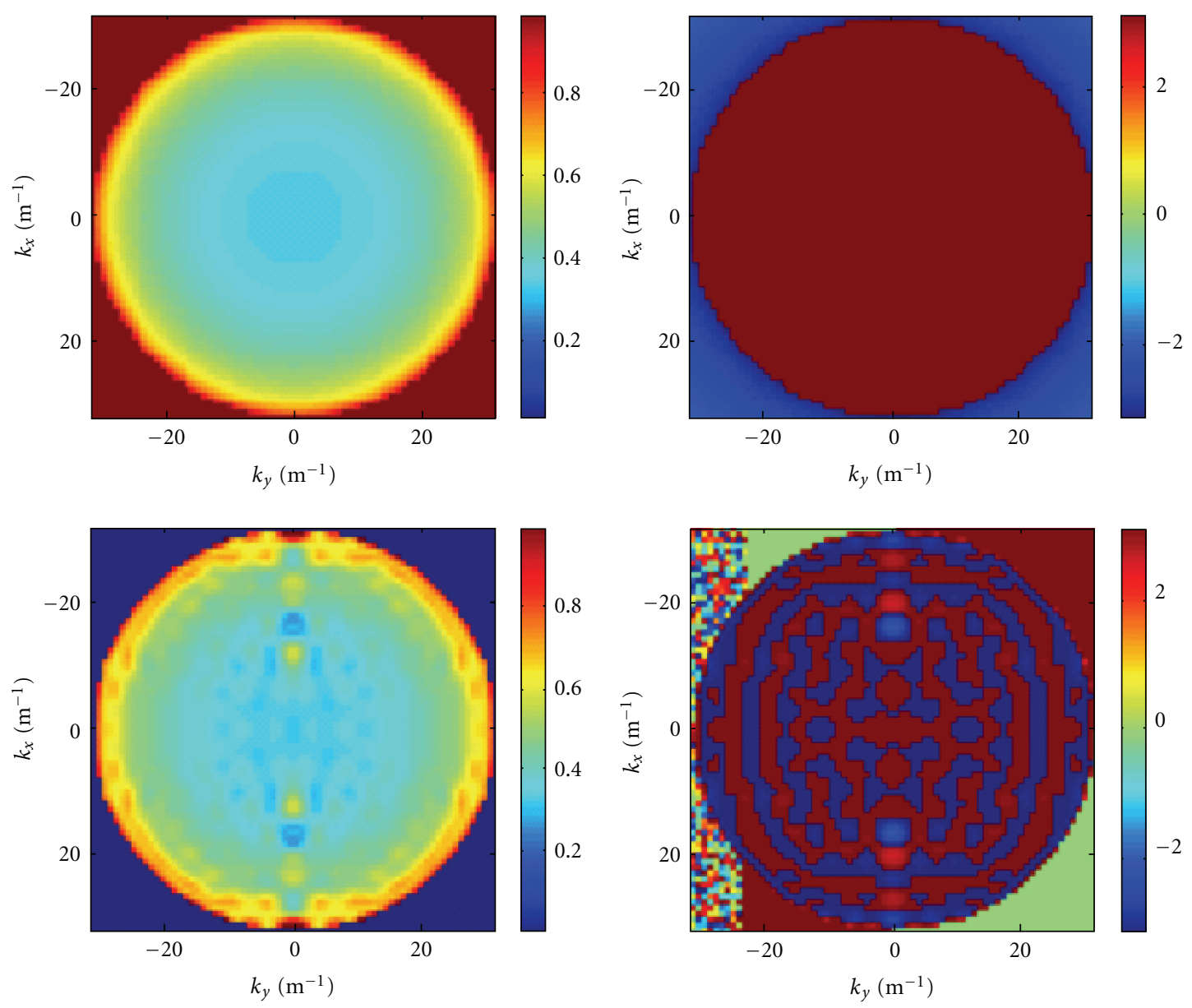

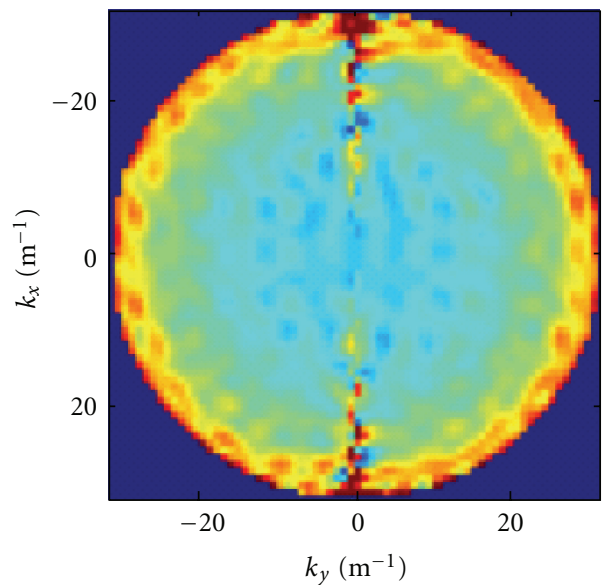

(a)

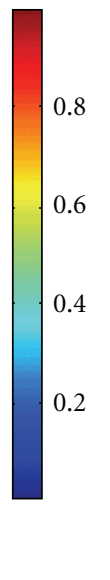

$6 \stackrel{\overbrace{}}{\stackrel{7}{g}}$

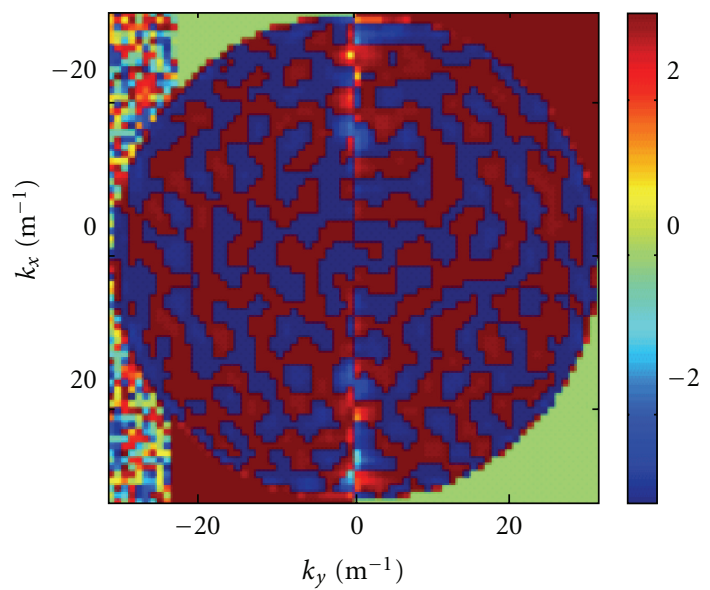

(b)

Figure 4: Soil 1. Amplitude (a) and phase (b) of $\Gamma_{\mathrm{TE}}$. Top line: actual values; middle line: noiseless estimation; bottom line: noisy estimation. 

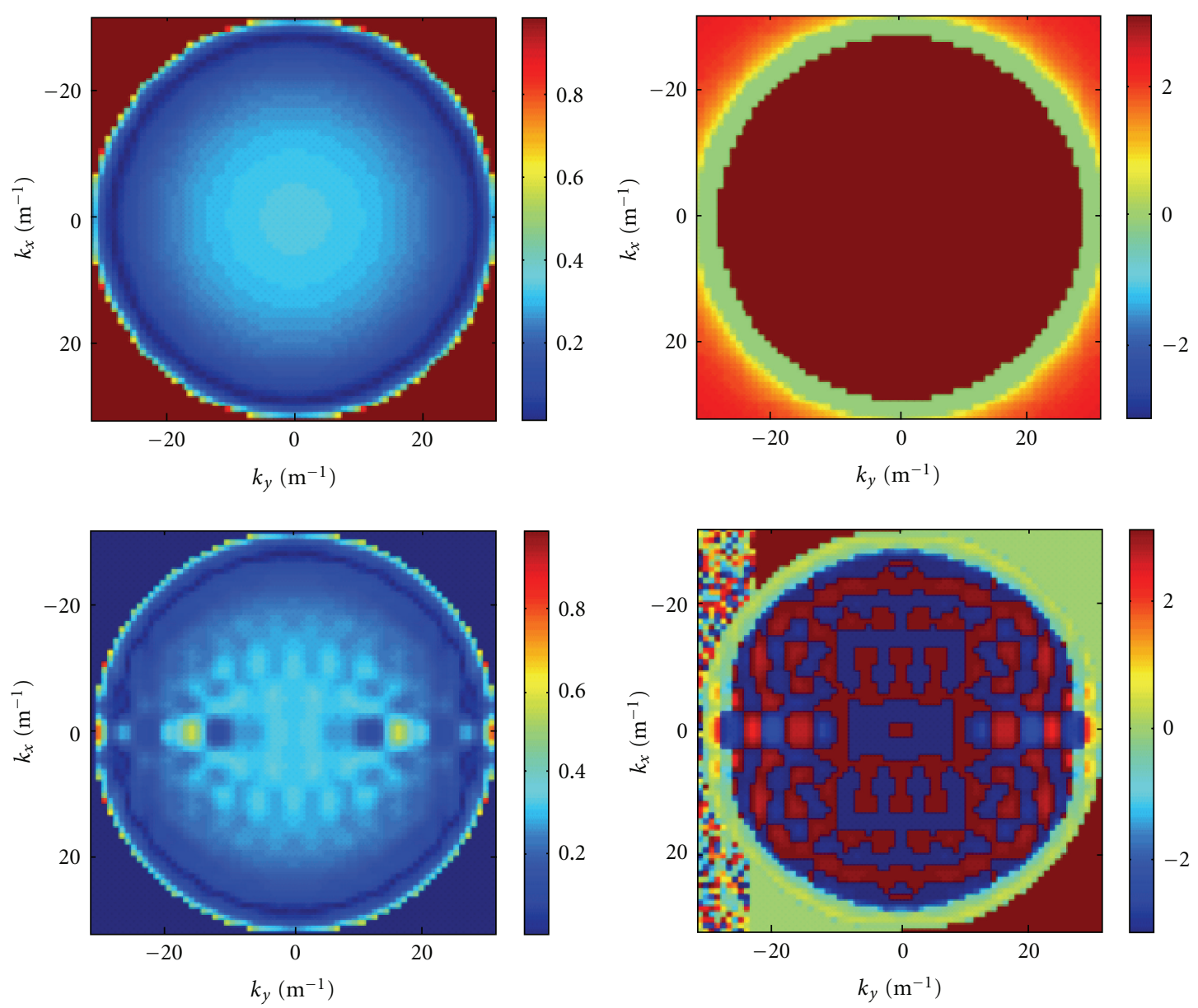

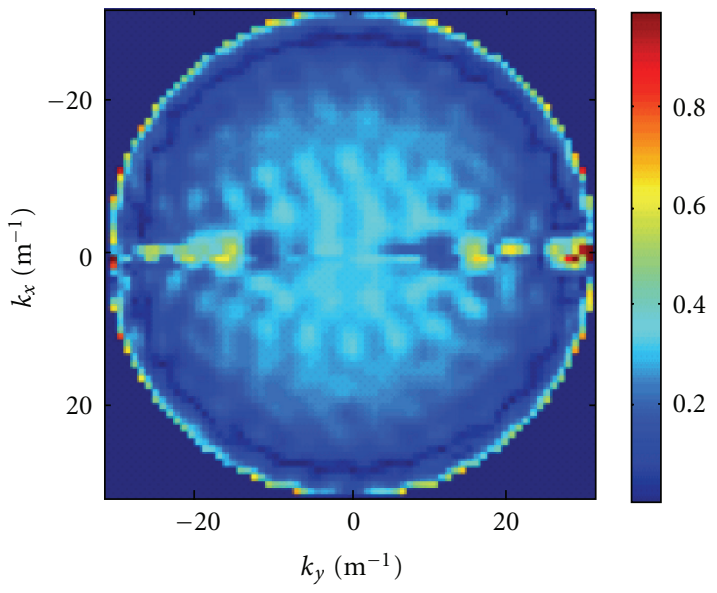

(a)

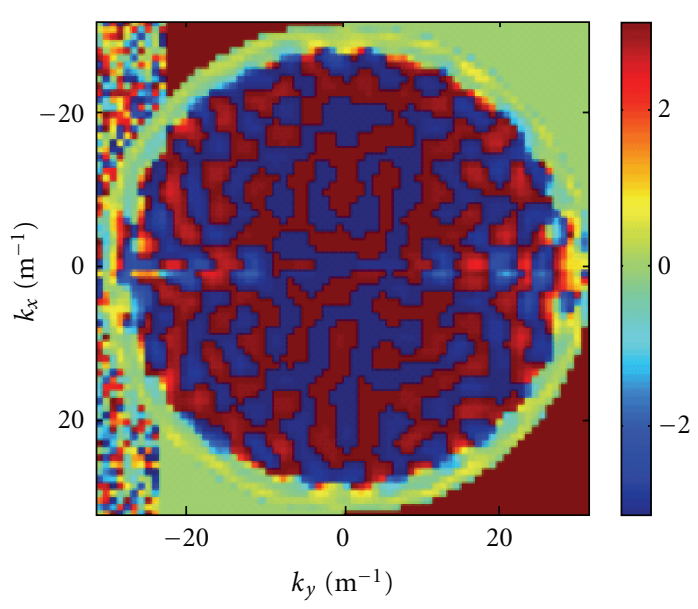

(b)

FIGURE 5: Soil 1. Amplitude (a) and phase (b) of $\Gamma_{\mathrm{TM}}$. Top line: actual values; middle line: noiseless estimation; bottom line: noisy estimation. 

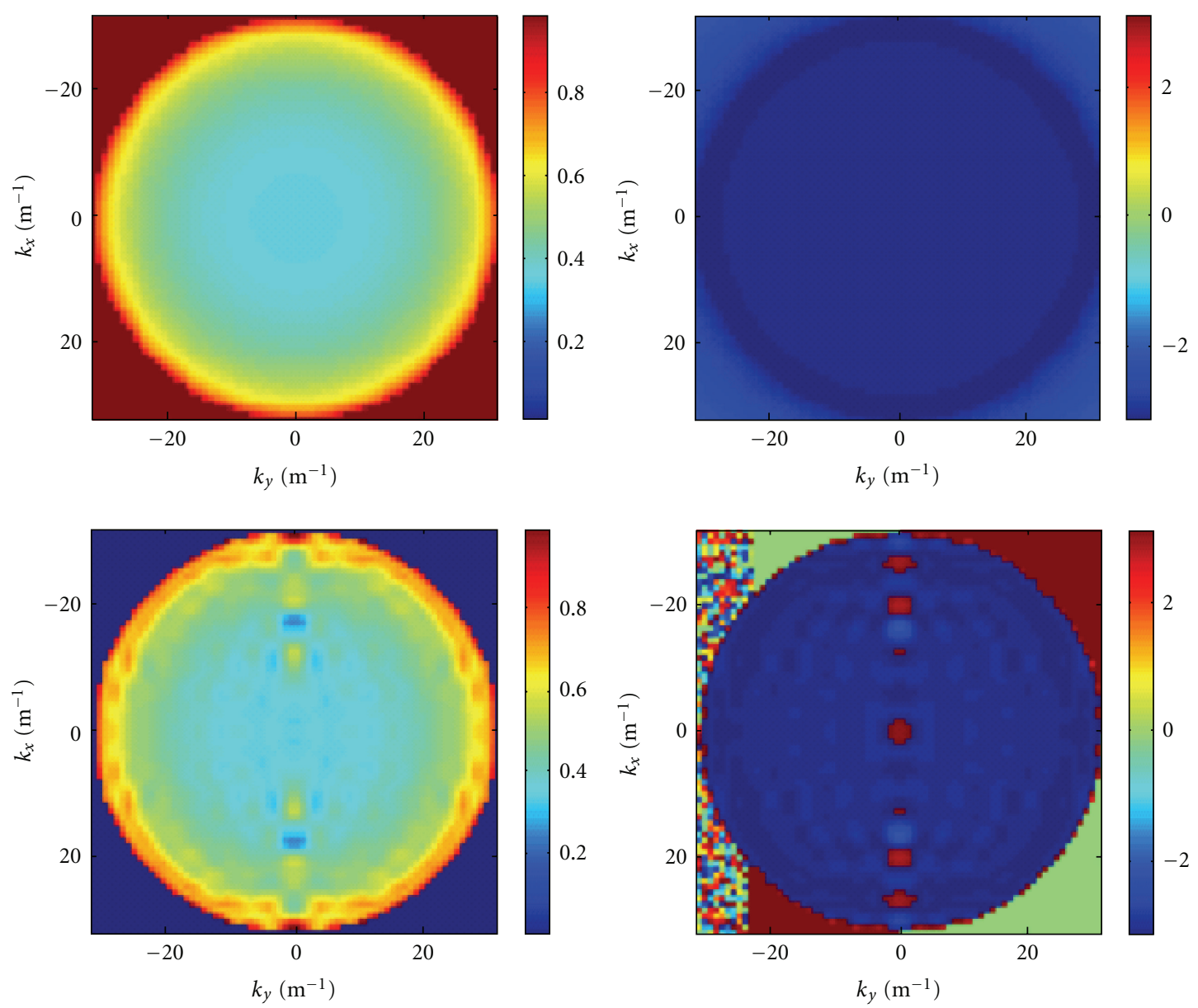

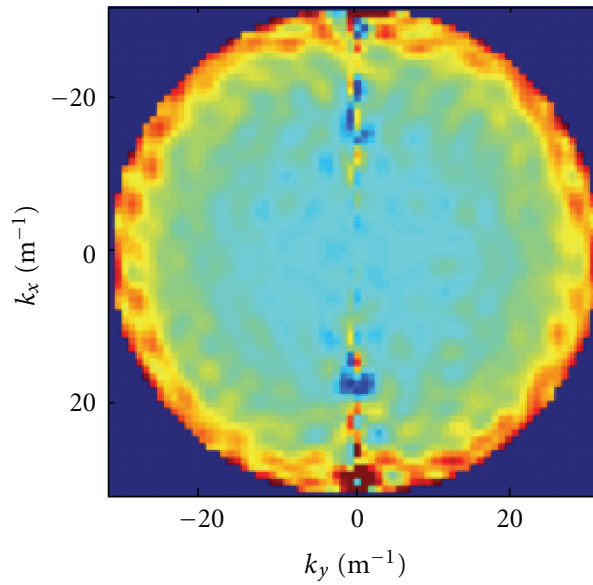

(a)

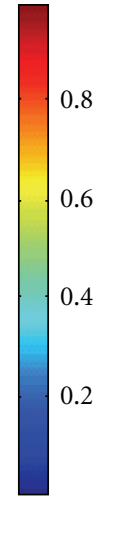

6

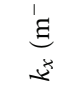

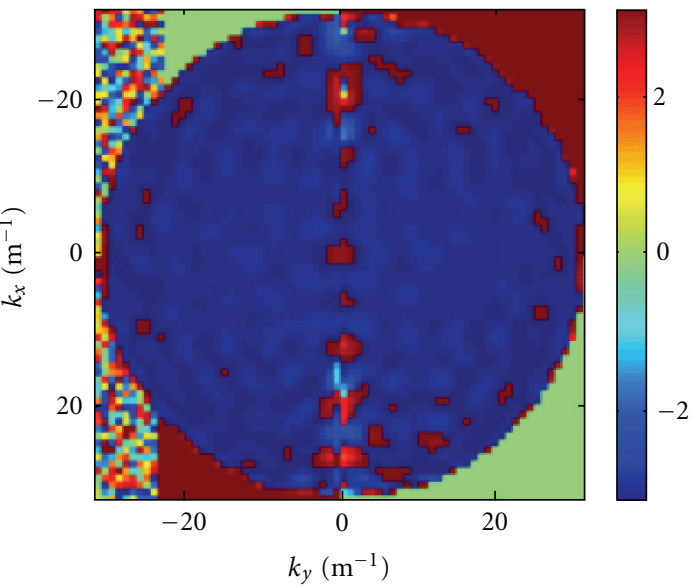

(b)

FIgure 6: Soil 2. Amplitude (a) and phase (b) of $\Gamma_{\mathrm{TE}}$. Top line: actual values; middle line: noiseless estimation; bottom line: noisy estimation. 

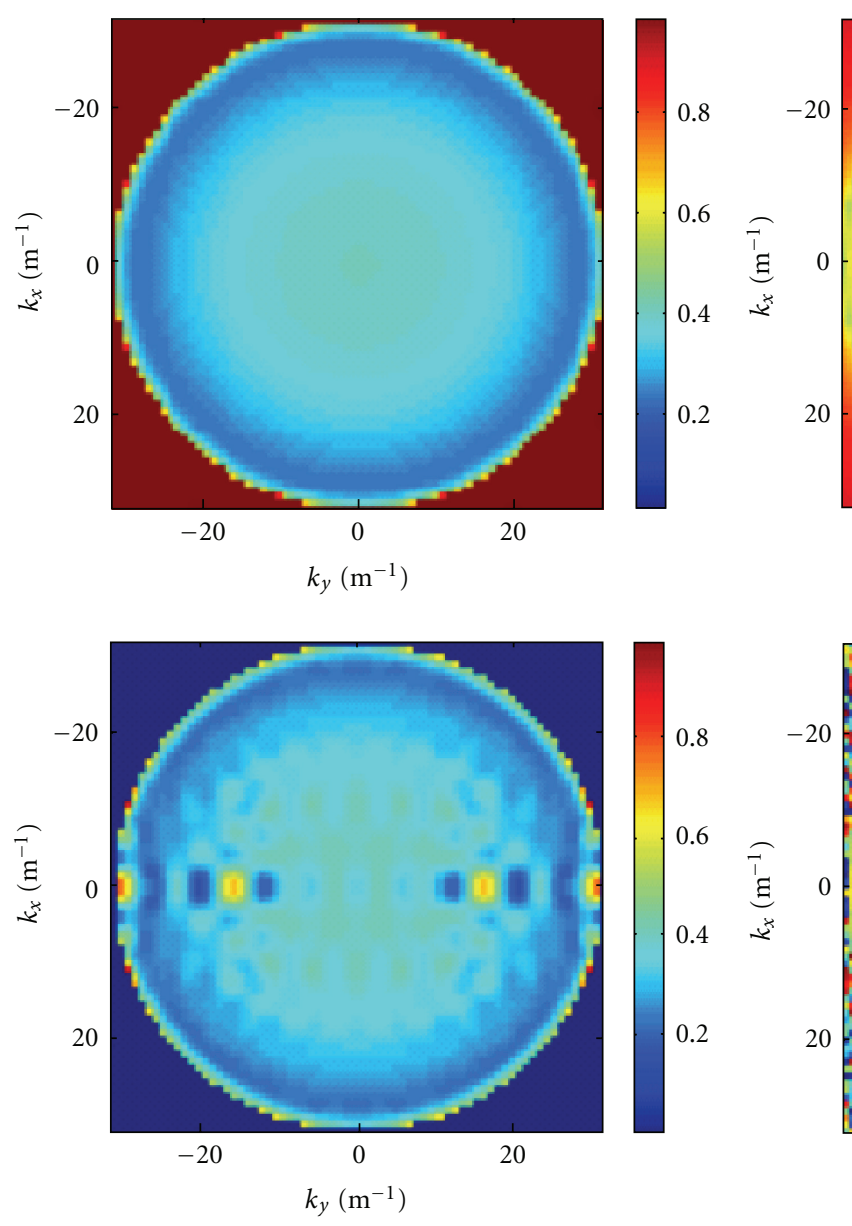

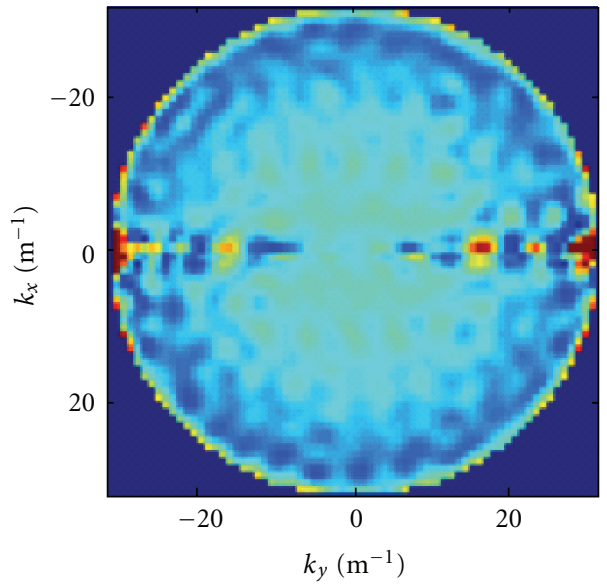

(a)
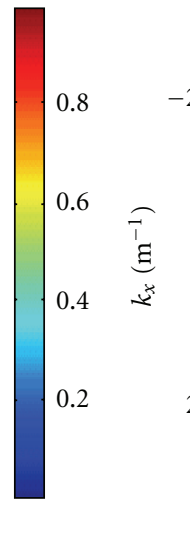

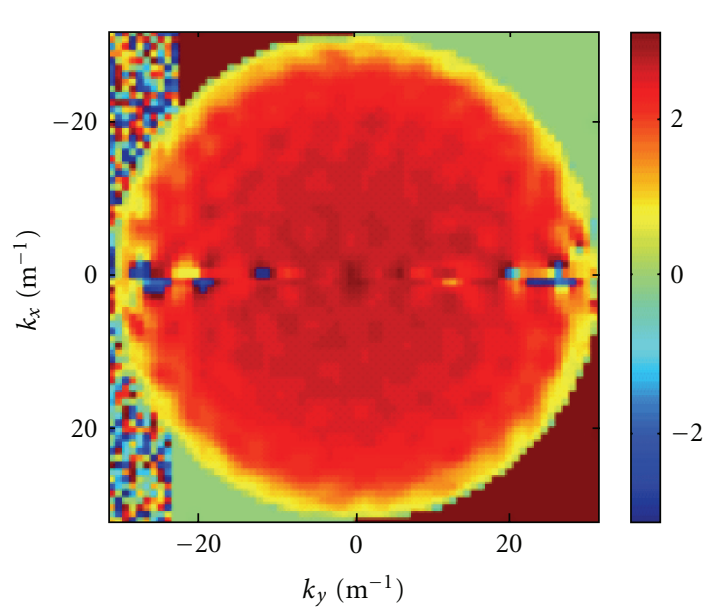

(b)

Figure 7: Soil 2. Amplitude (a) and phase (b) of $\Gamma_{\mathrm{TM}}$. Top line: actual values; middle line: noiseless estimation; bottom line: noisy estimation. 

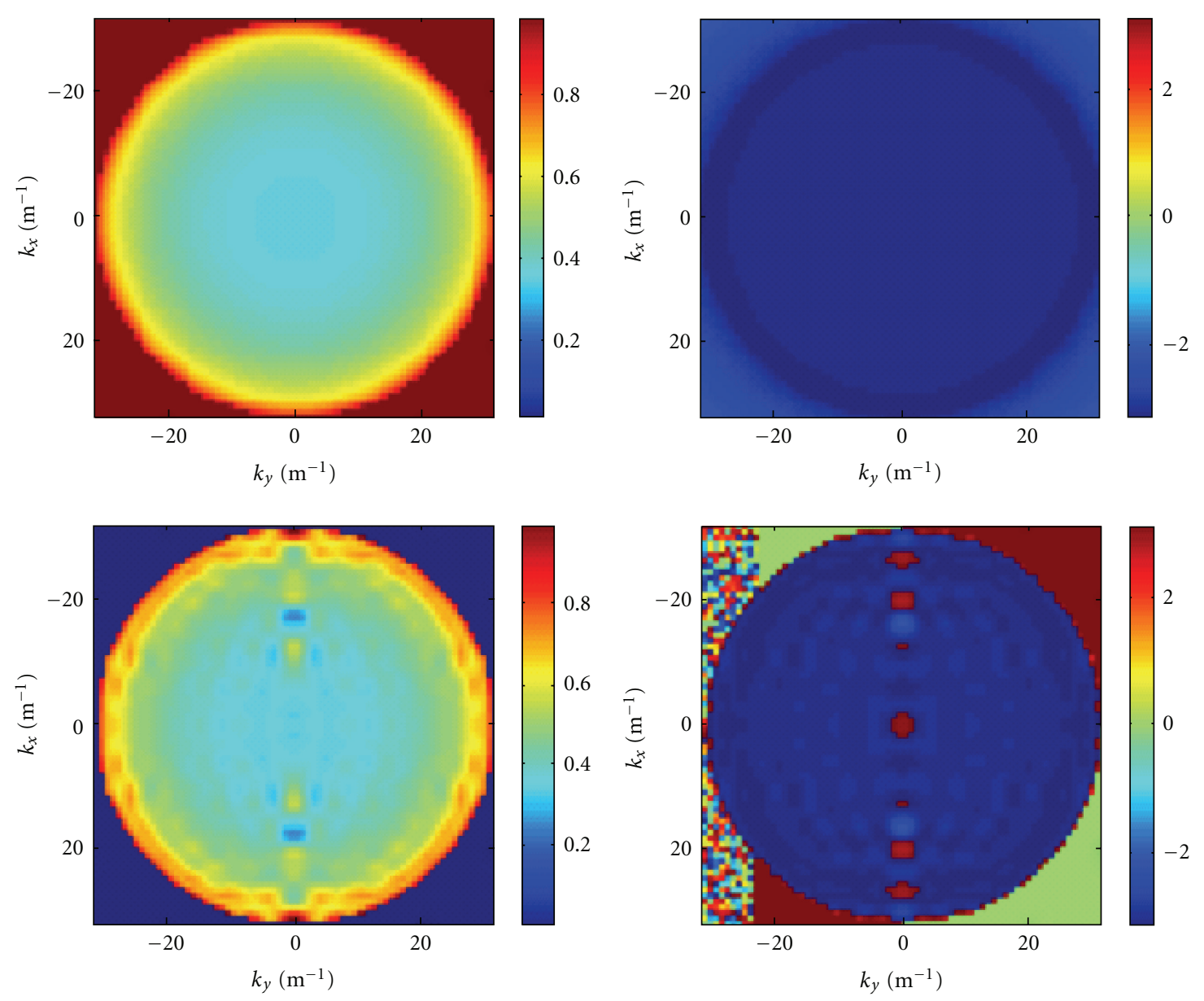

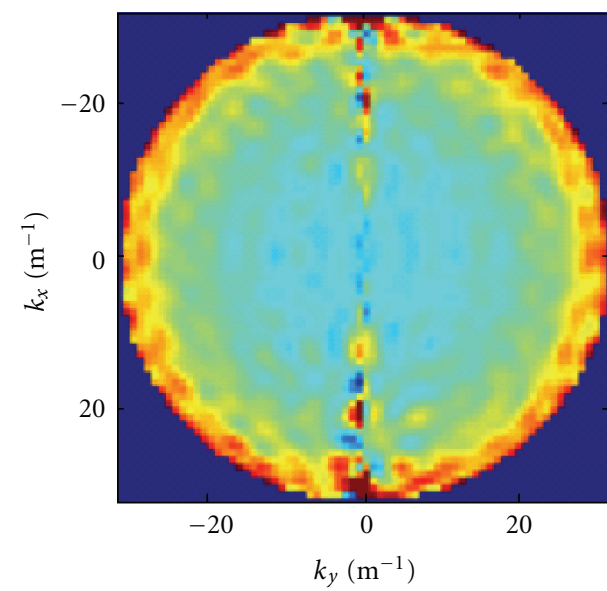

(a)

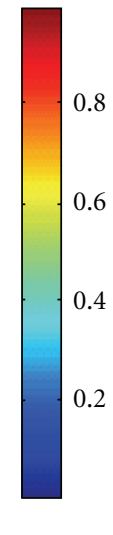

0.6

I

0.2

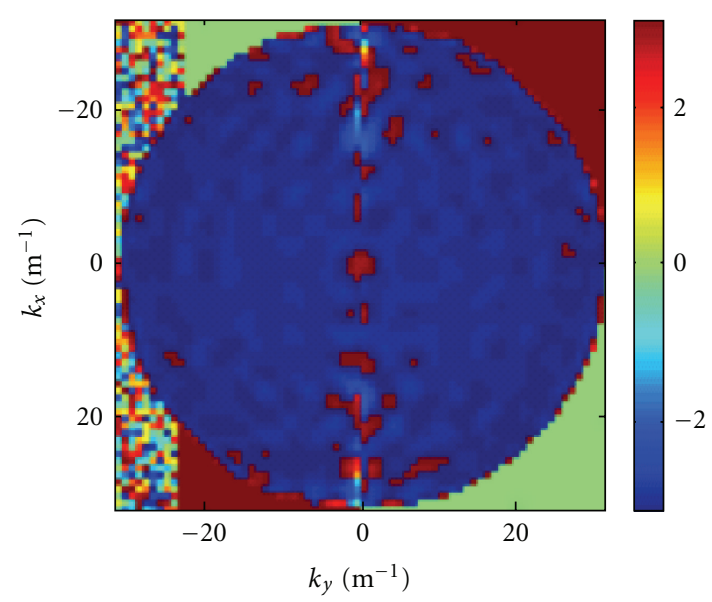

(b)

FIGURE 8: Soil 3. Amplitude (a) and phase (b) of $\Gamma_{\mathrm{TE}}$. Top line: actual values; middle line: noiseless estimation; bottom line: noisy estimation. 

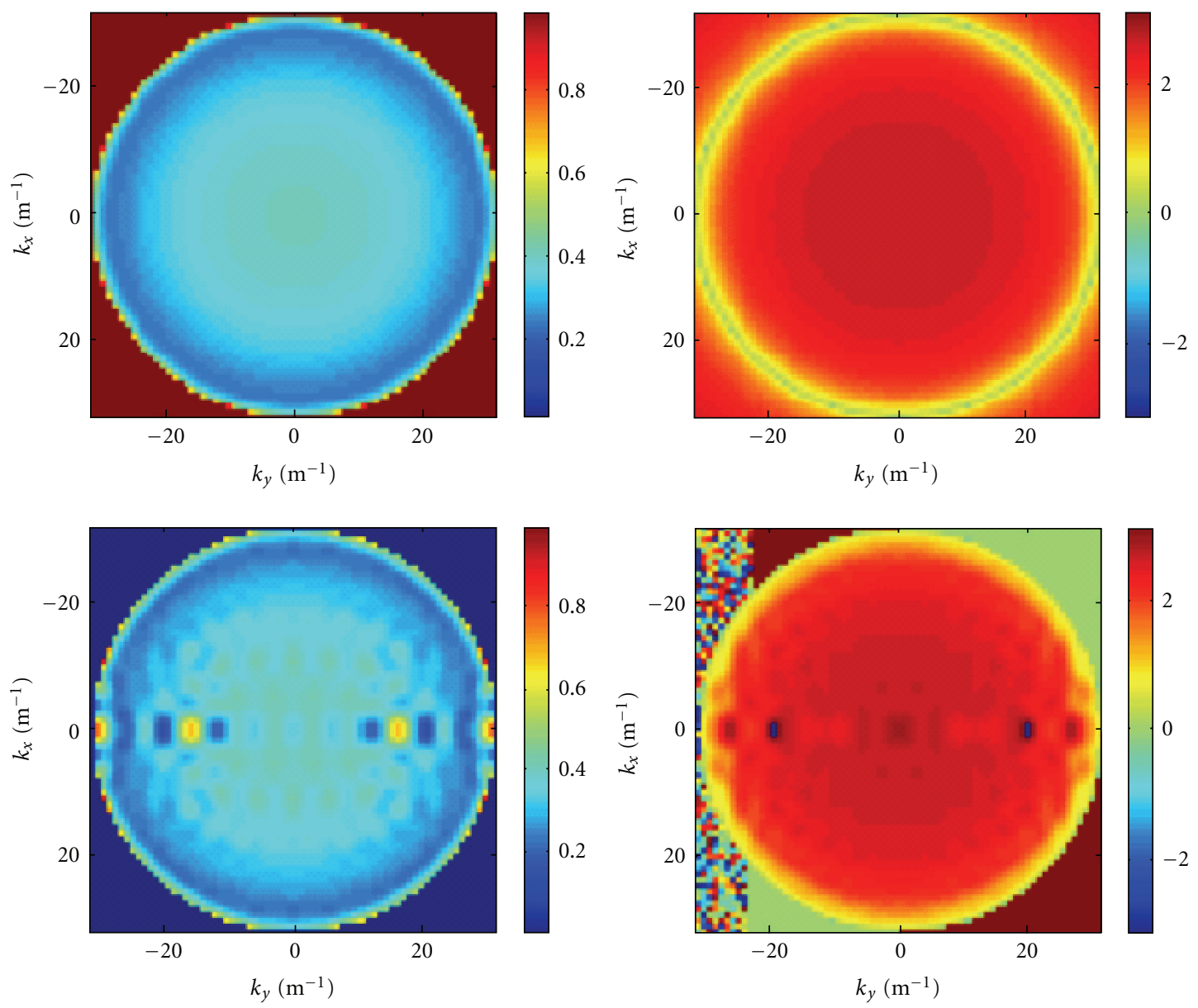

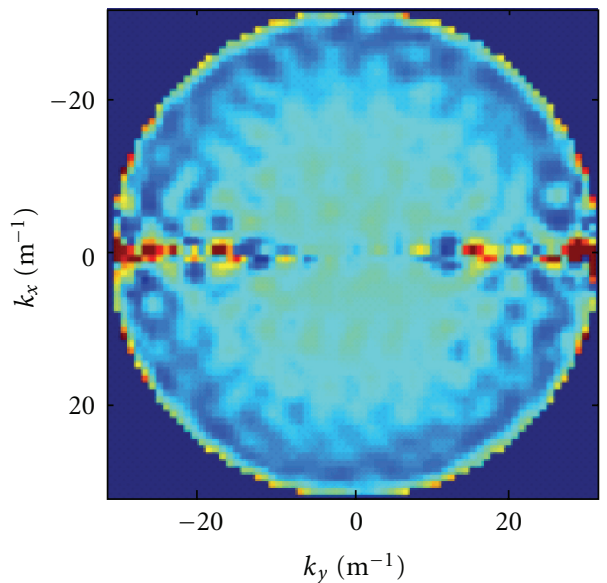

(a)

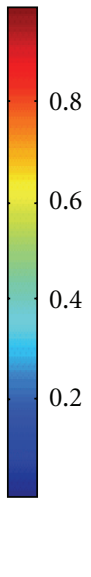

0.8
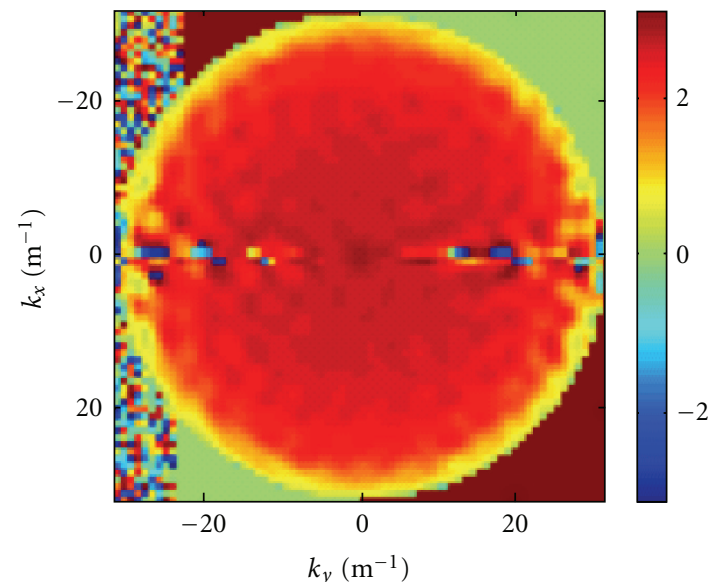

(b)

Figure 9: Soil 3. Amplitude (a) and phase (b) of $\Gamma_{\mathrm{TM}}$. Top line: actual values; middle line: noiseless estimation; bottom line: noisy estimation. 
By comparing actual and estimated coefficients, it can be noted that in all the cases estimation fails outside the visible domain whereas inside that domain some oscillations appear. This could be expected as they result from regularization, we applied through (9) to invert (6) and (7), which entails retrieving a filtered version of the unknown. By contrast, reconstructions appear rather "stable" against noise. More in detail, estimations fit well actual reflection coefficients expect along the lines at $k_{y}=0$ for $\Gamma_{\mathrm{TE}}$ and $k_{x}=0$ for $\Gamma_{\mathrm{TM}}$. This is due to the "numerical" singularities that (8) introduce while passing from the retrieved scattering parameters $\hat{\Gamma}_{11}(\cdot)$ and $\hat{\Gamma}_{21}(\cdot)$ to the Fresnel coefficients. However, this drawback can be circumvented by getting rid estimation along the "singular" lines and replacing such values by some interpolation scheme.

\section{Conclusions}

In this contribution, we extended our previous work concerning the Fresnel reflection coefficients estimation from reflected field data [13] by including also the role of the receiving antenna.

It is shown that, even in the case of homogeneous soil, the corresponding mathematical problem is rather interesting as it amounts to solving a vector linear integral equation where the unknowns, the Fresnel coefficients, appear embodied within a dyadic term. To solve such a problem a twostep procedure is used. First, a pair of half-space scattering parameters are determined by inverting two integral equations by means of the TSVD scheme. Then, Fresnel reflection coefficients are easily estimated by algebraic equations.

The method allows to retrieve the Fresnel coefficients as function of the angle of incidence (spatial harmonics); therefore it can be used to build up Green's function which is what is really necessary to obtain focalized subsurface images. The estimation procedure must be run for each adopted frequency. By contrast soil dispersive laws are not required to be a priori known.

Numerical results are used to show the effectiveness of estimation proposed procedure for different and typical kinds of soil. Except for certain numerical singularities, estimation's fits well the actual reflection coefficient within the visible domain, which in turn generally is prominent contribution with respect to the evanescent waves.

\section{References}

[1] D. Daniels, Ground Penetrating Radar, IEE Press, London, UK, 2nd edition, 2004.

[2] F. Soldovieri and R. Solimene, "Ground penetrating radar subsurface imaging of buried objects," in Radar Technology, G. Kouemou, Ed., In-Tech, 2010.

[3] D. A. Robinson, "Measurement of the solid dielectric permittivity of clay minerals and granular samples using a time-domain reflectometry immersion method," Vadose Zone Journal, vol. 3, no. 2, pp. 705-713, 2004.

[4] A. P. Annan, Ground Penetrating Radar Workshop Notes, Sensors \& Software, Mississauga, Canada, 2001.
[5] X. Zeng, G. A. McMechan, and T. Xu, "Synthesis of amplitudeversus-offset variations in ground-penetrating radar data," Geophysics, vol. 65, no. 1, pp. 113-125, 2000.

[6] J. Deparis and S. Garambois, "On the use of dispersive APVO GPR curves for thin-bed properties estimation: theory and application to fracture characterization," Geophysics, vol. 74, no. 1, pp. J1-J12, 2009.

[7] F. Soldovieri, G. Prisco, and R. Persico, "A strategy for the determination of the dielectric permittivity of a lossy soil exploiting GPR surface measurements and a cooperative target," Journal of Applied Geophysics, vol. 67, no. 4, pp. 288295, 2009.

[8] S. Lambot, E. C. Slob, I. van den Bosch, B. Stockbroeckx, B. Scheers, and M. Vanclooster, "Estimating soil electric properties from monostatic ground-penetrating radar signal inversion in the frequency domain," Water Resources Research, vol. 40, no. 4, pp. W042051-W0420512, 2004.

[9] P. Meincke and T. B. Hansen, "Plane-wave characterization of antennas close to a planar interface," IEEE Transactions on Geoscience and Remote Sensing, vol. 42, no. 6, pp. 1222-1232, 2004.

[10] R. Solimene, F. Soldovieri, and A. D'Alterio, "Determination of the fresnel reflection coefficient of a half-space for medium estimation purposes," Progress in Electromagnetics Research B, no. 27, pp. 61-82, 2011.

[11] R. Persico, F. Soldovieri, and G. Leone, "A microwave tomographic imaging approach for multibistatic configuration: the choice of the frequency step," IEEE Transactions on Instrumentation and Measurement, vol. 55, no. 6, pp. 19261934, 2006.

[12] R. Solimene, F. Ahmad, and F. Soldovieri, "A novel CS-TSVD strategy to perform data reduction in linear inverse scattering problems," IEEE Geoscience and Remote Sensing Letters, vol. 9, no. 5, pp. 881-885, 2012.

[13] R. Solimene, A. D'Alterio, and F. Soldovieri, “Determining Fresnel reflection coefficients in 3D half-space geometry by GPR multistatic data," Near Surface Geophysics, vol. 9, no. 3, pp. 265-275, 2011.

[14] R. Solimene, A. D'Alterio, and F. Soldovieri, "Half-space estimation by time gating based strategy," in Proceedings of the 13th Internarional Conference on Ground Penetrating Radar (GPR '10), pp. 683-687, Lecce, Italy, June 2010.

[15] R. E. Collin, Antennas and Radiowave Propagation, McGrawHill, New York, NY, USA, 1985.

[16] M. Bertero and P. Boccacci, Introduction to Inverse Problems in Imaging, IOP, Bristol, UK, 1998. 

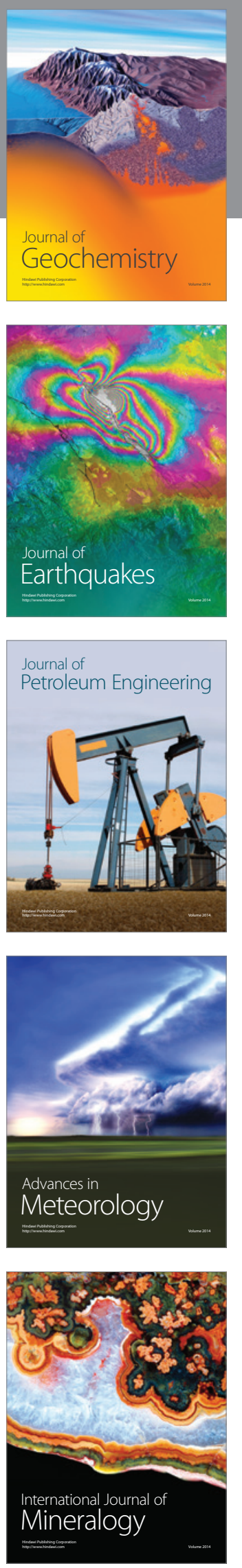
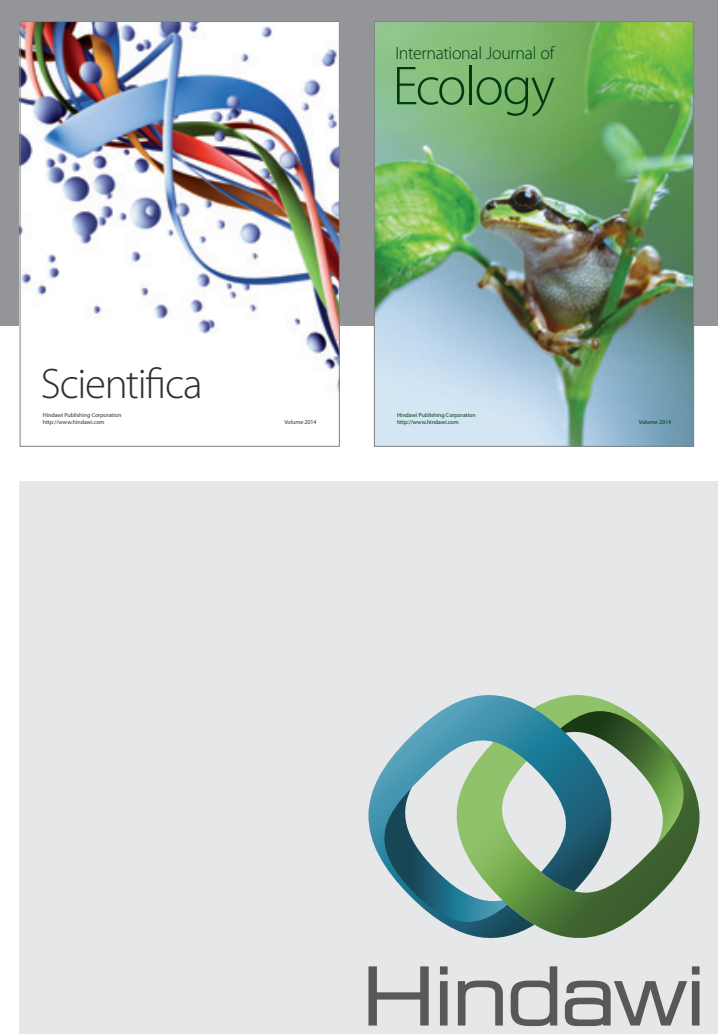

Submit your manuscripts at http://www.hindawi.com
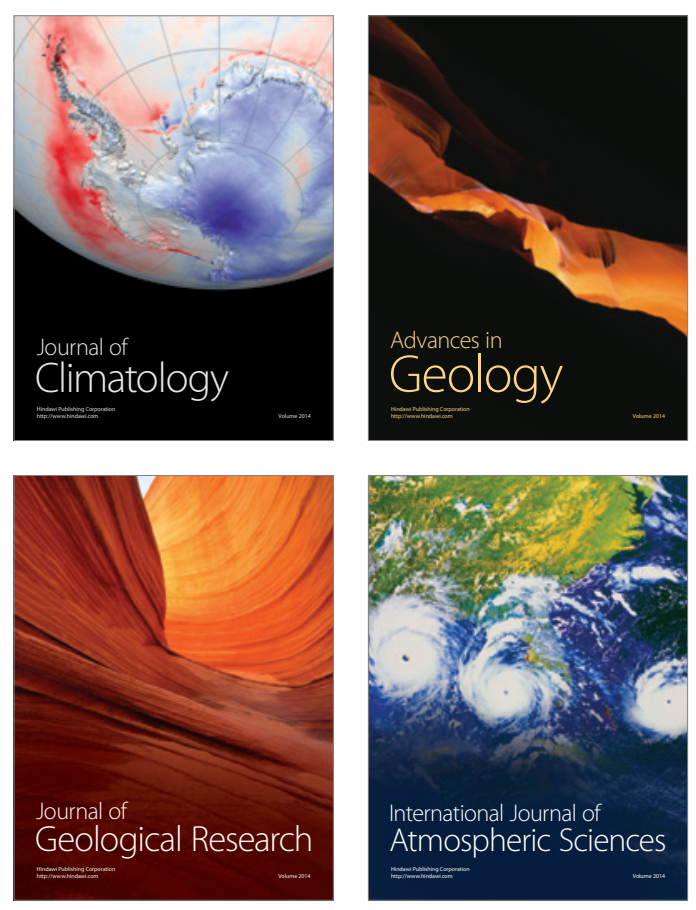
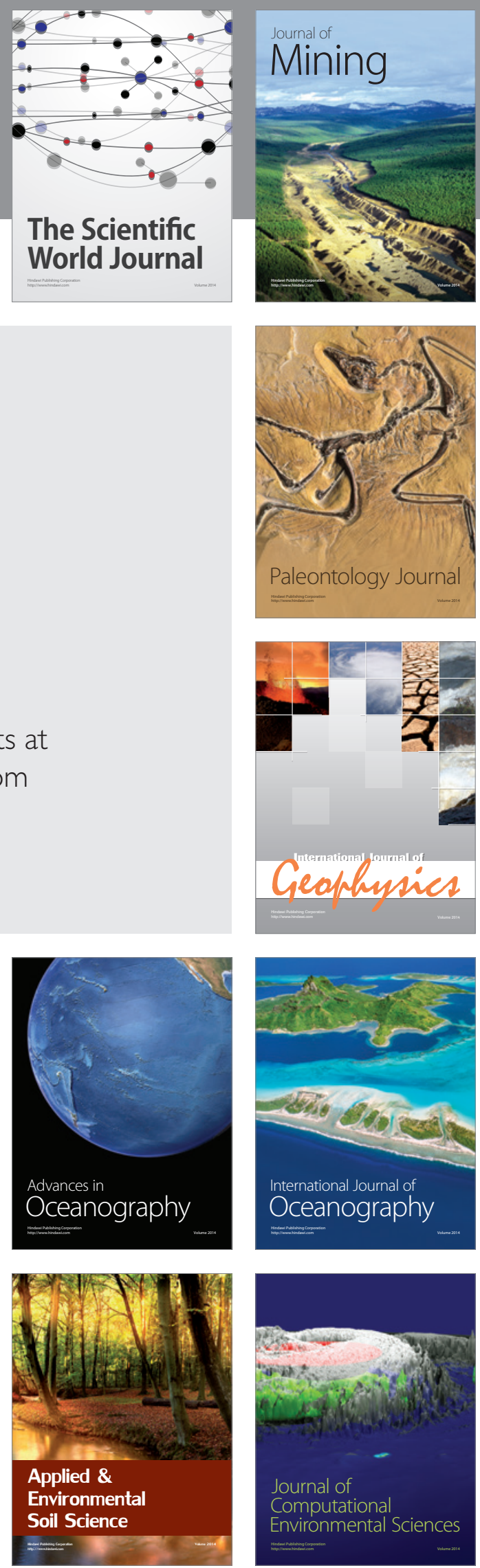